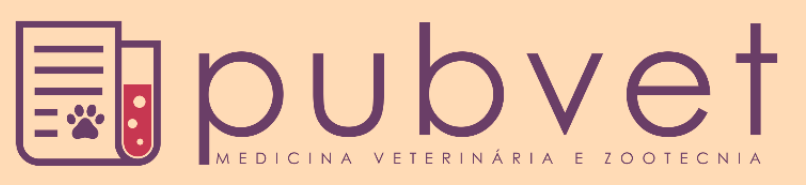

https://doi.org/10.31533/pubvet.v13n2a274.1-10

\title{
Toxoplasma gondii: possível mediador de alterações comportamentais em humanos
}

\author{
Mariana Fachini Esperança ${ }^{1 *} \bullet$, Dielson da Silva Vieira ${ }^{2} \bullet$, Welber Daniel Zanetti Lopes $^{3 \bullet}$, Dreyf \\ de Assis Gonçalves $^{4}{ }^{\circ}$, Katia Denise Saraiva Bresciani5 ${ }^{\circ}$, Weslen Fabricio Pires Teixeira ${ }^{6}{ }^{\circ}$ \\ ${ }^{1}$ Professora da Universidade Paulista- Unip, Instituto de Ciências Humanas. Araçatuba-SP, Brasil. \\ ${ }^{2}$ Doutorando em Ciência Animal pela Universidade Estadual Paulista Júlio de Mesquita Filho, Departamento de Clínica, Cirurgia e \\ Reprodução Animal, Araçatuba-SP, Brasil. \\ ${ }^{3}$ Professor da Universidade Federal de Goiás, Instituto de Patologia Tropical e Saúde Pública, Goiânia-GO, Brasil. \\ ${ }^{4}$ Doutorando em Ciência Animal pela Universidade Estadual Paulista Júlio de Mesquita Filho, Departamento de Apoio, Produção e Saúde \\ Animal, Araçatuba-SP, Brasil. \\ ${ }^{5}$ Professora da Universidade Estadual Paulista Júlio de Mesquita Filho, Departamento de Apoio, Produção e Saúde Animal, Araçatuba-SP, Brasil. \\ ${ }^{6}$ Professor Orientador da Universidade Federal de Goiás, Departamento de pós-graduação em Veterinária, Goiânia-GO, Brasil. \\ *Autor para correspondência. E-mail: fachiniesperanca@gmail.com.
}

Resumo. O objetivo deste estudo foi realizar uma revisão de literatura sobre o papel do $T$. gondii na mediação de alterações comportamentais em humanos. O T. gondii é um protozoário intracelular responsável pela infecção de diferentes espécies de aves e mamíferos, incluindo os homens. Os felídeos são os únicos hospedeiros definitivos deste parasito, sendo os gatos domésticos considerados os principais representantes na manutenção do ciclo deste protozoário em ambiente urbano. A infecção em humanos pode ocorrer por ingestão de cistos teciduais presentes em carne crua, ingestão de oocistos esporulados ou por via congênita. Diferentes estudos atribuíram ao T. gondii a capacidade de mediar mudanças de comportamento em animais e humanos. Em estudos realizados com humanos infectados foram observadas alterações de personalidade e redução no tempo de resposta aos estímulos ambientais. Embora, muitos estudos apontem para a existência de modificações comportamentais mediadas pelo $T$. gondii em seres humanos, tais inferências ainda não são totalmente compreendidas, sendo necessárias mais pesquisas com metodologias que permitam conclusões definitivas sobre o tema.

Palavras-chave: coccídeo, parasito, personalidade, psicologia

\section{Toxoplasma gondii: possible mediator of behavioral changes in humans}

\begin{abstract}
The objective of this study was to conduct a literature review on the role of $T$. gondii in mediating behavioral changes in humans. T. gondii is an intracellular protozoan responsible for the infection of different species of birds and mammals, including humans. The felids are the only definitive hosts of this parasite, and domestic cats are considered the main representatives in the maintenance of the cycle of this protozoan in an urban environment. Infection in humans can occur through ingestion of tissue cysts present in raw meat, ingestion of sporulated or congenital oocysts. Different studies attributed to $T$. gondii the ability to mediate behavioral changes in animals and humans. In studies with infected humans, personality changes and reduction of response time to environmental stimuli were observed. Although many studies point to the existence of behavioral changes mediated by $T$. gondii in humans, such inferences are not yet fully understood, and further research is needed with methodologies that allow definitive conclusions on the subject.
\end{abstract}

Keywords: coccyx, parasite, personality, psychology 


\section{Toxoplasma gondii: posible mediador del comportamiento cambios en los seres humanos}

Resumen. El objetivo de este estudio fue realizar una revisión de literatura sobre el papel
del T. gondii en la mediación de alteraciones comportamentales en humanos. El T. gondii
es un protozoario intracelular responsable de la infección de diferentes especies de aves y
mamíferos, incluidos los hombres. Los felinos son los únicos anfitriones definitivos de este
parásito, siendo los gatos domésticos considerados los principales representantes en el
mantenimiento del ciclo de este protozoario en ambiente urbano. La infección en humanos
puede ocurrir por ingestión de quistes en tejidos presentes en carne cruda, ingestión de
oocistos esporulados o por vía congénita. Diferentes estudios atribuyeron al T. gondii la
capacidad de mediar cambios de comportamiento en animales y humanos. En estudios
realizados con humanos infectados se observaron alteraciones de personalidad y reducción
en el tiempo de respuesta a los estímulos ambientales. Aunque muchos estudios apuntan a
la existencia de modificaciones comportamentales mediadas por el T. gondii en seres
humanos, tales inferencias aún no son totalmente comprendidas, siendo necesarias más
investigaciones con metodologías que permitan conclusiones definitivas sobre el tema.

Palabras clave: coccídeo, parásito, personalidad, psicología

\section{Introdução}

As causas de doenças crônicas e progressivas (doenças Parkinson e a demência de Alzheimer), dos transtornos de humor e ansiedade (Transtorno Bipolar e Transtorno Obsessivo-Compulsivo), doenças psiquiátricas (esquizofrenia) e dos distúrbios neurológicos graves não infecciosos (epilepsias) são estudadas há anos por médicos e psicólogos do mundo inteiro. Apesar de muitos estudos apontarem diversos fatores predisponentes, tais como: heranças genéticas, estresse, traumas emocionais e psicológicos, fatores biológicos e sociais, até o presente momento, nenhum estudo apresentou um consenso científico da etiologia dessas doenças mentais (Fontenelle \& Mendlowicz, 2017). Nesse sentido, pesquisadores da área de medicina veterinária vêm realizando diferentes estudos que apontaram a ocorrência de alterações comportamentais em animais e humanos, possivelmente mediadas pela infecção por Toxoplasma gondii (Dentillo, 2013).

O T. gondii é um protozoário intracelular obrigatório de distribuição cosmopolita capaz de parasitar aves e mamíferos, incluindo humanos (Dubey, 2010). Os felídeos são particularmente importantes na perpetuação do ciclo biológico deste coccídeo, por serem considerados os únicos hospedeiros definitivos deste parasito (Dubey et al., 2004). Apenas nos felídeos é completado o ciclo enteroepitelial do T. gondii, levando a formação e excreção de oocistos nas fezes, que contaminam o ambiente, proporcionando a disseminação deste parasito entre outras espécies animais (Dubey, 1998).

Em humanos a infecção pelo $T$. gondii pode ocorrer principalmente por meio da ingestão de oocistos esporulados presentes na água ou alimentos contaminados ou pela ingestão de cistos teciduais presentes em tecidos de hospedeiros intermediários portadores da infecção crônica pelo parasito (Dubey, 2010). A transmissão transplacentária é outra importante via de disseminação da toxoplasmose entre humanos, que ocorre geralmente quando uma gestante é primoinfectada pelo parasito durante o terço final da gestação (Dubey \& Towle, 1986; Mamidi et al., 2002).

Segundo Yan et al. (2016) fatores referentes à transmissão e distribuição da infecção toxoplásmica estão diretamente correlacionados ao impacto ecológico provocado pelos humanos e seus hábitos alimentares e higiênicos. A toxoplasmose humana geralmente se manifesta na forma assintomática; sendo o surgimento de sinais clínicos mais comuns em hospedeiros imunocomprometidos (principalmente portadores da imunodeficiência adquirida), podendo evoluir para encefalite toxoplásmica e morte Pereira-Chioccola et al. (2009). A infecção em humanos pode levar ao surgimento de má formação fetal, natimortos, aborto espontâneo, cegueira congênita, hidrocefalia, retardo mental e a perda parcial ou total da visão (Galvan-Ramírez et al., 2012).

Diferentes estudos indicam que infecções crônicas com a presença de cistos cerebrais do T. gondii, estão provavelmente envolvidas no surgimento de transtornos psiquiátricos em seres humanos 
discutindo a capacidade deste coccídeo em mediar alterações comportamentais em indivíduos parasitados (Fekadu et al., 2010; Parlog et al., 2015). Este artigo aborda diferentes resultados e discussões sobre a possível atuação do T. gondii na mediação de alterações comportamentais em humanos infectados.

\section{Material e métodos}

Foi realizado levantamento na base de dados periódicos CAPES, na área de Medicina Veterinária, nas bases de dados Scopus, Web of Science e Scielo. Utilizando como critério de inclusão artigos realizados em humanos e animais que correlacionaram a ocorrência de alterações de comportamentos com a toxoplasmose. O recorte temporal estabelecido para a seleção das publicações foi entre os anos de 1980 e 2018. Foi aplicado o filtro de idiomas para português, inglês e espanhol, nos tópicos: alterações comportamentais em humanos e Toxoplasma gondii.

\section{Resultados e discussão}

Cloninger et al. (1998), Flegr \& Hrdy (1994), Flegr et al. (2000), Flegr et al. (2003) e Novotná et al. (2005) realizaram testes de personalidades $16 \mathrm{~F}$ de Cattell e de Cloninger, encontrando diferenças de alterações comportamentais nos fatores de personalidade de indivíduos infectados e não infectados pelo T. gondii.

Homens infectados pelo parasito apresentam comportamentos mais propensos a ignorar as regras sociais, sendo, mais evasivos, inconvenientes e dissidentes. Também, são mais dogmáticos, desconfiados, ciumentos de acordo com os resultados dos fatores G e L (consciência e confiança) do teste 16PF de Cattell (Flegr \& Hrdy, 1994). Em contrapartida, sugere-se que mulheres infectadas são mais "calorosas" (afetivamente com outras pessoas), extrovertidas, conscientes as regras sociais e culturais, persistentes e moralistas segundo os fatores A e G no 16PF de Cattell (expansividade e consciência) (Flegr et al., 2000). Quando comparados a indivíduos não infectados, tanto mulheres quanto os homens infectados apresentaram aumento no fator $\mathrm{O}$ de Cattell (apreensão), apresentando comportamentos mais inseguros, apreensivos e perturbados durante a infecção toxoplásmica (Flegr \& Hrdy, 1994; Flegr et al., 2000).

Quanto ao fator Q3 do 16PF (disciplina e organização), os homens infectados apresentaram resultados mais baixos do que os não infectados (Flegr \& Hrdy, 1994) e as mulheres infectadas, resultados mais altos do que as não infectadas. Níveis baixos no fator Q3 estão relacionados com comportamentos de desorganização, em que, o indivíduo relata sentir-se bem em ambientes desorganizados. Os resultados altos dizem respeitos aos comportamentos de organização, em que, o indivíduo se sente confortável quando planeja cada etapa de suas ações e pode apresentar dificuldade em lidar com a imprevisibilidade (Novotná et al., 2005). Em relação ao fator A do 16 PF (Expansividade), os homens infectados obtiveram resultados mais baixos do que os homens não infectados, apresentando comportamentos mais reservados, tendendo a gostar de trabalhar sozinhos, frequentemente em atividades mecânicas, intelectuais ou artísticas. Já em mulheres, não foi observada para esse fator, diferença significativa entre o grupo infectado e não infectado (Novotná et al., 2005).

Por meio do teste Cloninger TCI foi observado que homens e mulheres positivos para toxoplasmose apresentaram diminuição do fator "busca por novidade" (Flegr et al., 2003; Novotná et al., 2005) corroborando com Cloninger et al. (1998), que observaram que indivíduos com baixos escores deste fator, são descritos como pouco irritadiços, reflexivos, controlados, reservados, tolerantes à monotonia e pouco entusiasmados com coisas novas, permanecendo submetidos a situações pouco estimulantes e pouco recompensadoras por longos períodos. Diminuição do tempo de reação e déficit de atenção foi os encontrado mais evidentes em doadores de sangue portadores assintomáticos da toxoplasmose (Alvarado-Esquivel et al., 2012; Flegr et al., 2002; Havlíček et al., 2001; Yereli et al., 2006). Segundo Havlíček et al. (2001) e Flegr et al. (2002), tais alterações psicomotoras podem estar envolvidas diretamente no papel da infecção toxoplásmica em aumentar a ocorrência de acidentes no trânsito e no trabalho. Reforçando essa inferência, em um estudo realizado na República Checa, amostras sorológicas de condutores de automóveis e pedestres acidentados foram colhidas e analisadas quanto à presença de anticorpos contra $T$. gondii. A soro prevalência diagnosticada sugeriu que indivíduos infectados com o parasito têm 2,65 vezes mais risco de sofrer acidentes de trânsito que pessoas não infectadas (Alvarado- 
Esquivel et al., 2012). Segundo Cook et al. (2015), humanos infectados por T. gondii possuem uma menor capacidade de regulação dos impulsos primários, desenvolvendo traços de agressividade, impulsividade e aumentando os comportamentos violentos e de risco entre eles.

A ocorrência de anticorpos anti-T. gondii em homicidas (Flegr et al., 2014; Lester, 2010, 2012), presidiários (Alvarado-Esquivel et al., 2014) e suicidas (Arling et al., 2009; Ling et al., 2011) foram evidenciadas em diferentes estudos que relataram a correlacão positiva entre a infecção toxoplásmica e o comportamento das pessoas avaliadas. Segundo Pedersen et al. (2012), mães infectadas com o $T$. gondii apresentam maior risco de violência auto-dirigida e suicídio que mães não infectadas, fato também observado por Ling et al. (2011) que verificaram a correlação positiva entre a infecção por $T$. gondii e a ocorrência de suicídio em mulheres com idade pós-menopausa.

Há divergências nos estudos encontrados em relação à influência ou não do Toxoplasma na etioliga do Transtorno Obsessivo Compulsivo (TOC). O TOC é uma doença psiquiátrica considerada relativamente comum, que atinge cerca de $2 \%$ a $3 \%$ das pessoas ao longo da vida (Crino et al., 2005). As manifestações do transtorno acontecem por meio de obsessões e compulsões, em que o sujeito repete padrões de comportamentos de ansiedade, sentindo-se cada dia mais frustrado e estressado em decorrência do tempo que leva para completar sua compulsão e os resultados que essa interferência temporal tem sobre seu funcionamento social, acadêmico ou profissional (Sales et al., 2010). Resultados obtidos por Memik et al. (2015) em indivíduos de 9 a 18 anos de idade, diagnosticados com TOC, não correlacionaram a infecção por Toxoplasma a ocorrência desta psicopatologia, diagnosticando a soropositividade de anticorpos ( $\mathrm{IgG}$ ) anti-T. gondii em 4,8\% das crianças com TOC e em 8,9\% das crianças pertencentes ao grupo controle (sem TOC).

Em contra partida, estudos de Miman et al. (2010a) e Brynska et al. (2001) evidenciam a correlação positiva entre a infecção por $T$. gondii com a ocorrência do TOC. No primeiro estudo, as manifestações crônicas do TOC foram relacionadas à toxoplasmose adquirida durante a infância ou adolescência. Este estudo foi realizado com dois pacientes diagnosticados com TOC na infância (um menino de 14 e o outro de 11 anos). Para ambos os casos, os exames laboratoriais confirmaram o diagnóstico de toxoplasmose, em que, a terapia farmacológica da infecção por $T$. gondii sem qualquer tratamento psicofarmacológico causou remissão ou melhora significativa dos sintomas do TOC (Brynska et al., 2001). No estudo de Miman et al. (2010b) foram selecionados 42 pacientes com TOC e 100 indivíduos saudáveis com faixa etária entre 18 e 79 anos, sendo investigada a associação entre a infecção por Toxoplasma e o TOC por meio da taxa de soro-positividade para anticorpos anti-Toxoplasma (IgG) obtidas por meio do ELISA. Os níveis de IgG entre os pacientes com TOC $(47,62 \%)$ foi maior que entre os pacientes saudáveis (19\%).

Divergências etiológicas sobre a ocorrência da doença de Parkinson (DP) em relação à influência pelo T. gondii também foram observadas. A DP é uma enfermidade neurodegenerativa progressiva crônica, caracterizada por disfunções motoras e cognitivas (Kamei et al., 2010). Com o progresso desta enfermidade, o indivíduo vai comprometendo as funções cognitivas e executivas, apresentando inicialmente tremores nos membros superiores, em especial nas mãos e dedos que evoluem para o restante do corpo ao decorrer do tempo (Macuglia et al., 2012). Miman et al. (2010a) demonstraram uma correlação entre a infecção toxoplásmica com os mecanismos patogênicos da DP. Nesse estudo foram utilizados 52 pacientes diagnosticados com DP seguindo os critérios do United Kingdom Parkinson's disease Society Brain Bank Clinical Diagnostic e 40 indivíduos saudáveis. Os resultados positivos para IgG apresentaram diferenças entre os grupos experimentais, sendo diagnosticada a infecção por T. gondii em 42,3\% (22/52) dos indivíduos com DP e 22,5\% (9/40) dos pacientes sem a doença. Tais autores sugeriram que as degenerações dos neurônios produtores de dopamina observada nessa enfermidade podem estar relacionadas com a produção de dopamina pelo $T$. gondii. Em contrapartida, Oskouei et al. (2016a) não observaram qualquer associação estatística entre a infecção toxoplásmica e a ocorrência da doença de Parkinson, em um estudo realizado no Irã. Neste estudo foram avaliadas as ocorrências de anticorpos (IgG) anti-T. gondii em dois grupos experimentais (indivíduos com DP e sem DP) de 75 indivíduos com idade média de 63 anos, não sendo observada diferença na ocorrência de anticorpos (IgG) anti-T. gondii entre o grupo de indivíduos com e sem DP (ocorrências de $85,0 \%$ e $90,3 \%$, respectivamente). 
A doença de Alzheimer (DA) é uma alteração neurodegenerativa progressiva que resulta em perda irreversível de neurônios, responsável por aproximadamente dois terços de todos os casos de demência mundial (Bruns, 2009). Pelo fato do $T$ gondii ser reconhecido como importante agente infeccioso envolvido em doenças neurodegenerativas (Dalimi \& Abdoli, 2012), pesquisas que buscam correlaciona-lo com a ocorrência da DA vem sendo realizadas. Diferenças entre os níveis séricos de IgG dosados em indivíduos com DA e indivíduos sem a doença foram observados por Kusbeci et al. (2011). Neste estudo foram utilizados 34 pacientes com DA (grupo controle: indivíduos positivos para DA) e 37 indivíduos saudáveis (grupo experimental: indivíduos negativos para DA), com idade média de 72 anos, que não apresentavam diferenças socioeconômicas. A ocorrência de anticorpos (IgG) anti-T. gondii nos indivíduos com DA foi de $44,1 \%$, ocorrência estatisticamente superior a diagnosticada no grupo de indivíduos sem DA (24,3\%), sugerindo que a infecção toxoplásmica pode estar correlacionada com os mecanismos patogênicos dessa neurodegeneração. Resultados divergentes foram observados por Oskouei et al. (2016b). Neste estudo foram utilizados 150 indivíduos distribuídos em dois grupos experimentais (75 indivíduos sem DA e 75 indivíduos com DA), não sendo foi observada diferença entre a ocorrência de anticorpos (IgG) contra $T$. gondii nos indivíduos sem DA $(62,6 \%)$ e com a doença $(61,3 \%)$.

O Transtorno Bipolar (TB) é uma psicopatologia de humor considerada complexa, grave, crônica, com alta herdabilidade (Ledford, 2011). Os prejuízos na funcionalidade global se dão pelas alterações patológicas de humor, caracterizadas por episódios de remissão e recidiva de depressão e mania/hipomaníaca (Kapczinski et al., 2016). A etiologia dessa enfermidade ainda é desconhecida, sendo a sua correlação com a infecção toxoplásmica, pouco estudada. Na França, ao analisar 110 pacientes com TB e 106 pacientes saudáveis, Hamdani et al. (2013) observaram que a soropositividade para T. gondii foi superior nos indivíduos com TB (76,9\%) que nos saudáveis $(48,2 \%)$. Embora os referidos autores tenham indicado cautela na interpretação dos resultados obtidos devido ao número amostral utilizado, eles confirmaram a associação positiva entre a soropositividade para $T$. gondii e a ocorrência do transtorno bipolar. Associação positiva entre a ocorrência de transtorno bipolar, perda cognitiva e a infecção por $T$. gondii foi também relatada (Hamdani et al., 2015). Neste estudo foram observadas maiores concentrações de IL-6 (duas vezes superior) nos indivíduos infectados por T. gondii, levando a crer que a longa exposição à inflamação, medida com a expressão da IL-6 nos pacientes com TB e infectados pelo T. gondii, pode levar a alterações das funções cognitivas do indivíduo (Hamdani et al., 2015).

A epilepsia é caracterizada por distúrbios neurológicos que causam crises epilépticas em aproximadamente $1 \%$ da população mundial. Esta enfermidade é dividida em Epilepsia Idiopática com origem na infância e com a etiologia desconhecida e em Epilepsia Criptogênica que se origina de lesões cerebrais causadas por trauma ou de origem infecciosa (Fisher et al., 2014). No estudo de Critchley et al. (1982) foram pesquisadas por meio da técnica de ELISA, a ocorrência de anticorpos anti $T$. gondii em amostras sorológicas de 40 crianças com epilepsia criptogênica, 30 com epilepsia idiopática e 20 crianças saudáveis. Os níveis de $\operatorname{IgG}$ anti $T$. gondii foi maior entre crianças com epilepsia criptogênica (20\%) do que nas portadoras de epilepsia idiopática (0\%) e saudáveis (10\%).

Segundo Prandovszky et al. (2011) o aumento nos níveis de dopamina em roedores com toxoplasmose pode ser explicado pelo fato do parasito mediar à síntese de L-DOPA que é precursor de dopamina. Tal correlação tem sido defendida principalmente devido ao aumento nos níveis de dopamina diagnosticadas em casos de esquizofrenia humana e durante a infecção toxoplásmica (Diehl \& Gershon, 1992; Howes \& Kapur, 2009; Torrey \& Yolken, 2003), transtorno obsessivo compulsivo, transtorno bipolar e em suicidas (Berk et al., 2007; Denys et al., 2004; Diehl \& Gershon, 1992; Roy et al., 1992)

A esquizofrenia é um distúrbio neuropsiquiátrico complexo, crônico e debilitante. Os sintomas mais comuns são: alucinações, delírios, emoções embotadas, pensamento desordenado e sem nexo com a realidade (Carter et al., 2008). Por apresentar etiologia desconhecida, fatores etiológicos como a infecção por T. gondii, são descritos como causadores desta doença (Flegr, 2007; Jones-Brando et al., 2003; Mortensen et al., 2007; Niebuhr et al., 2008; Torrey et al., 2006; Torrey et al., 2012; Torrey et al., 2000; Torrey \& Yolken, 2003; Yolken et al., 2001; Yolken \& Torrey, 2008). A ocorrência de esquizofrenia vem sendo diretamente correlacionada ao desequilíbrio nas concentrações de dopamina entre as regiões mesolímbica e mesocortical do cérebro (Sawa \& Snyder, 2002), o que segundo Flegr 
(2007) poderia explicar a associação observada entre casos desta neuropsicose e da infecção toxoplasmose. Outra evidência desta associação foi o fato de alguns medicamentos antipsicóticos e estabilizadosres do humor utilizados na terapêutica de humanos com esquizofrenia inibirem a replicação, invasão e possivelmente a "manipulação" comportamental exercida pelo T. gondii em roedores, impedindo que estes animais desenvolvessem a atração suicida por felinos (Skallova et al., 2006; Webster et al., 2006).

A hipótese de manipulação comportamental do T. gondii sob seus hospedeiros vem sendo muito defendida por diferentes autores. Flegr (2007) sugere que a infecção crônica pelo parasito pode influenciar o comportamento não só de roedores parasitados, como também de seres humanos, sendo os mecanismos neurofisiológicos e os efeitos destas mudanças comportamentais, ainda não elucidados completamente. De acordo com Berdoy et al. (2000) tal hipótese de manipulação comportamental pode estar associada a mecanismos de perpetuação de espécie exercidos pelo parasito, onde ele busque maneiras de aumentar as chances de entrar em contato com um hospedeiro definitivo felino e completar seu ciclo de vida. Um exemplo clássico que sugere esta hipótese é o fato de camundongos com toxoplasmose perderem a aversão ao cheiro de urina de gatos podendo assim aumentar as chances destes roedores serem predados pelos hospedeiros definitivos do parasito.

Segundo Flegr (2007), o aumento de acidentes de trânsito e tentativas de suicídio entre os humanos infectados pelo parasito pode exemplificar a atuação contemporânea deste protozoário na manipulação do comportamento. A atuação do T. gondii sob o comportamento humano é um tema considerado fascinante e igualmente polêmico entre a comunidade científica. É importante relatar que em alguns estudos realizados sobre o tema não foram observadas correlações positivas entre a infecção pelo parasito e o surgimento de alterações comportamentais em humanos (Memik et al., 2015; Oskouei et al., 2016a; Oskouei et al., 2016b). Segundo Sugden et al. (2016) os dados existentes na literatura consultada não suportam a hipótese de que a infecção por $T$. gondii está relacionada com alterações comportamentais negativas em parte representativa da população humana.

\section{Considerações finais}

Estudos que avaliaram a correlação da infecção toxoplásmica com a ocorrência de alterações comportamentais em humanos estão sendo amplamente divulgados na literatura mundial nas últimas décadas. Pela ausência de metodologias que permitam o controle de todos os fatores que possam, por ventura, estar influenciando na ocorrência tanto das alterações comportamentais como do $T$. gondii em humanos, os estudos aqui apresentados ainda provocam muita polêmica dentro da comunidade cientifica, sendo necessária a realização de mais estudos que permitirão melhor fundamentar a hipótese de modulação comportamental por este protozoário.

\section{Referências bibliográficas}

Alvarado-Esquivel, C., Hernández-Tinoco, J., Sánchez-Anguiano, L. F., Ramos-Nevárez, A., CerrilloSoto, S. M., Sáenz-Soto, L. \& Liesenfeld, O. (2014). High seroprevalence of Toxoplasma gondii infection in inmates: a case control study in Durango City, Mexico. European Journal of Microbiology and Immunology, 4(1):76-82.

Alvarado-Esquivel, C., Torres-Castorena, A., Liesenfeld, O., Estrada-Martínez, S. \& Urbina-Álvarez, J. D. (2012). High seroprevalence of Toxoplasma gondii infection in a subset of Mexican patients with work accidents and low socioeconomic status. Parasites \& Vectors, 5(1):13.

Arling, T. A., Yolken, R. H., Lapidus, M., Langenberg, P., Dickerson, F. B., Zimmerman, S. A., . . . Tonelli, L. H. (2009). Toxoplasma gondii antibody titers and history of suicide attempts in patients with recurrent mood disorders. The Journal of Nervous and Mental Disease, 197(12):905-908.

Berdoy, M., Webster, J. P. \& Macdonald, D. W. (2000). Fatal attraction in rats infected with Toxoplasma gondii. Proceedings of the Royal Society of London. Series B: Biological Sciences, 267(1452):15911594.

Berk, M., Dodd, S., Kauer-Sant'anna, M., Malhi, G. S., Bourin, M., Kapczinski, F. \& Norman, T. (2007). Dopamine dysregulation syndrome: implications for a dopamine hypothesis of bipolar disorder. Acta Psychiatrica Scandinavica, 11641-49. 
Bruns, A. I. (2009). Alzheimer's disease. Brit Med J; 338: 464-471. British Medical Journal, 338464 471.

Brynska, A., Tomaszewicz-Libudzic, E. \& Wolanczyk, T. (2001). Obsessive-compulsive disorder and acquired toxoplasmosis in two children. European Child \& Adolescent Psychiatry, 10(3):200-204.

Carter, C. S., Barch, D. M., Buchanan, R. W., Bullmore, E., Krystal, J. H., Cohen, J., . . Robbins, T. (2008). Identifying cognitive mechanisms targeted for treatment development in schizophrenia: an overview of the first meeting of the Cognitive Neuroscience Treatment Research to Improve Cognition in Schizophrenia Initiative. Biological Psychiatry, 64(1):4-10.

Cloninger, C. R., Svrakic, D. M. \& Przybeck, T. R. (1998). A psychobiological model of temperament and character. The Development of Psychiatry and its Complexity 975-990.

Cook, T. B., Brenner, L. A., Cloninger, C. R., Langenberg, P., Igbide, A., Giegling, I., . . Brundin, L. (2015). "Latent" infection with Toxoplasma gondii: association with trait aggression and impulsivity in healthy adults. Journal of Psychiatric Research, 6087-94.

Crino, R., Slade, T. \& Andrews, G. (2005). The changing prevalence and severity of obsessivecompulsive disorder criteria from DSM-III to DSM-IV. American Journal of Psychiatry, 162(5):876882.

Critchley, E. M. R., Vakil, S. D., Hutchinson, D. N. \& Taylor, P. (1982). Toxoplasma, toxocara, and epilepsy. Epilepsia, 23(3):315-321.

Dalimi, A. \& Abdoli, A. (2012). Latent toxoplasmosis and human. Iranian Journal of Parasitology, 7(1):1-17.

Dentillo, D. B. (2013). Toxoplasmose crônica pode manipular comportamento. Ciência e Cultura, 65(1):14-15.

Denys, D., Zohar, J. \& Westenberg, H. G. (2004). The role of dopamine in obsessive-compulsive disorder: preclinical and clinical evidence. The Journal of Clinical Psychiatry, 6511-17.

Diehl, D. J. \& Gershon, S. (1992). The role of dopamine in mood disorders. Comprehensive Psychiatry, 33(2):115-120.

Dubey, J. P. (1998). Advances in the life cycle of Toxoplasma gondii. International Journal for Parasitology, 28(7):1019-1024.

Dubey, J. P. (2010). Toxoplasmosis of Animals and Humans. Florida, USA: Boca Raton, CRC Press.

Dubey, J. P., Navarro, I. T., Sreekumar, C., Dahl, E., Freire, R. L., Kawabata, H. H., . . Thulliez, P. (2004). Toxoplasma gondii infections in cats from Paraná, Brazil: seroprevalence, tissue distribution, and biologic and genetic characterization of isolates. Journal of Parasitology, 90(4):721-726.

Dubey, J. P. \& Towle, A. (1986). Toxoplasmosis in sheep. Commonwealth Institute of Parasitology, 641-11.

Fekadu, A., Shibre, T. \& Cleare, A. J. (2010). Toxoplasmosis as a cause for behaviour disordersoverview of evidence and mechanisms. Folia Parasitologica, 57(2):105-113.

Fisher, R. S., Acevedo, C., Arzimanoglou, A., Bogacz, A., Cross, J. H., Elger, C. E., . . Glynn, M. (2014). ILAE official report: a practical clinical definition of epilepsy. Epilepsia, 55(4):475-482.

Flegr, J. (2007). Effects of Toxoplasma on human behavior. Schizophrenia Bulletin, 33(3):757-760.

Flegr, J., Havlícek, J., Kodym, P., Malý, M. \& Smahel, Z. (2002). Increased risk of traffic accidents in subjects with latent toxoplasmosis: a retrospective case-control study. BMC Infectious Diseases, 2(1):11.

Flegr, J. \& Hrdy, I. (1994). Influence of chronic toxoplasmosis on some human personality factors. Folia Parasitologica, 41(2):122-126.

Flegr, J., Kodym, P. \& Tolarová, V. (2000). Correlation of duration of latent Toxoplasma gondii infection with personality changes in women. Biological Psychology, 53(1):57-68.

Flegr, J., Prandota, J., Sovičková, M. \& Israili, Z. H. (2014). Toxoplasmosis-a global threat. Correlation of latent toxoplasmosis with specific disease burden in a set of 88 countries. PloS One, 9(3):e90203.

Flegr, J., Preiss, M., Klose, J., Havlíček, J., Vitáková, M. \& Kodym, P. (2003). Decreased level of psychobiological factor novelty seeking and lower intelligence in men latently infected with the 
protozoan parasite Toxoplasma gondii Dopamine, a missing link between schizophrenia and toxoplasmosis? Biological Psychology, 63(3):253-268.

Fontenelle, L. F. \& Mendlowicz, M. V. (2017). Manual de psicopatologia descritiva e semiologia psiquiátrica: Thieme Revinter Publicações LTDA.

Galvan-Ramírez, M. L., Troyo-Sanroman, R., Roman, S., Bernal-Redondo, R. \& Vázquez Castellanos, J. L. (2012). Prevalence of toxoplasma infection in Mexican newborns and children: a systematic review from 1954 to 2009. ISRN pediatrics, 20121-6.

Hamdani, N., Daban-Huard, C., Lajnef, M., Gadel, R., Le Corvoisier, P., Delavest, M., . . Houenou, J. (2015). Cognitive deterioration among bipolar disorder patients infected by Toxoplasma gondii is correlated to interleukin 6 levels. Journal of Affective Disorders, 179161-166.

Hamdani, N., Daban-Huard, C., Lajnef, M., Richard, J.-R., Delavest, M., Godin, O., . . Jamain, S. (2013). Relationship between Toxoplasma gondii infection and bipolar disorder in a French sample. Journal of Affective Disorders, 148(2-3):444-448.

Havlíček, J., Gašová, Z., Smith, A. P., Zvára, K. \& Flegr, J. (2001). Decrease of psychomotor performance in subjects with latent 'asymptomatic'toxoplasmosis. Parasitology, 122(5):515-520.

Howes, O. D. \& Kapur, S. (2009). The dopamine hypothesis of schizophrenia: version III-the final common pathway. Schizophrenia Bulletin, 35(3):549-562.

Jones-Brando, L., Torrey, E. F. \& Yolken, R. (2003). Drugs used in the treatment of schizophrenia and bipolar disorder inhibit the replication of Toxoplasma gondii. Schizophrenia Research, 62(3):237244.

Kamei, S., Morita, A., Serizawa, K., Mizutani, T. \& Hirayanagi, K. (2010). Quantitative EEG analysis of executive dysfunction in Parkinson disease. Journal of Clinical Neurophysiology, 27(3):193-197.

Kapczinski, N. S., Narvaez, J. C., Magalhães, P. V., Bücker, J., Peuker, A. C., Loredo, A. C., . . Fries, G. R. (2016). Cognition and functioning in bipolar depression. Brazilian Journal of Psychiatry, 38(3):201-206.

Kusbeci, O. Y., Miman, O., Yaman, M., Aktepe, O. C. \& Yazar, S. (2011). Could Toxoplasma gondii have any role in Alzheimer disease? Alzheimer Disease \& Associated Disorders, 25(1):1-3.

Ledford, H. (2011). Mental-health guide accused of overreach. Nature, 479(7372):163-163.

Lester, D. (2010). Brain parasites and suicide. Psychological Reports, 107(2):424-424.

Lester, D. (2012). Toxoplasma gondii and homicide. Psychological Reports, 111(1):196-197.

Ling, V. J., Lester, D., Mortensen, P. B., Langenberg, P. W. \& Postolache, T. T. (2011). Toxoplasma gondii seropositivity and suicide rates in women. The Journal of Nervous and Mental Disease, 199(7):440-444.

Macuglia, G. R., Rieder, C. R. M. \& Almeida, R. M. M. (2012). Funções executivas na doença de Parkinson: revisão da literatura. Psico, 43(4):552-561.

Mamidi, A., DeSimone, J. A. \& Pomerantz, R. J. (2002). Central nervous system infections in individuals with HIV-1 infection. Journal of Neurovirology, 8(3):158-167.

Memik, N. Ç., Tamer, G. S., Ünver, H. \& Gündoğdu, Ö. Y. (2015). The relationship between pediatric obsessive compulsive disorder and Toxoplasma gondii. Journal of Obsessive-Compulsive and Related Disorders, 724-28.

Miman, O., Kusbeci, O. Y., Aktepe, O. C. \& Cetinkaya, Z. (2010a). The probable relation between Toxoplasma gondii and Parkinson's disease. Neuroscience Letters, 475(3):129-131.

Miman, O., Mutlu, E. A., Ozcan, O., Atambay, M., Karlidag, R. \& Unal, S. (2010b). Is there any role of Toxoplasma gondii in the etiology of obsessive-compulsive disorder? Psychiatry Research, 177(1-2):263-265.

Mortensen, P. B., Nørgaard-Pedersen, B., Waltoft, B. L., Sørensen, T. L., Hougaard, D., Torrey, E. F. \& Yolken, R. H. (2007). Toxoplasma gondii as a risk factor for early-onset schizophrenia: analysis of filter paper blood samples obtained at birth. Biological Psychiatry, 61(5):688-693. 
Niebuhr, D. W., Millikan, A. M., Cowan, D. N., Yolken, R., Li, Y. \& Weber, N. S. (2008). Selected infectious agents and risk of schizophrenia among US military personnel. American Journal of Psychiatry, 165(1):99-106.

Novotná, M., Hanusova, J., Klose, J., Preiss, M., Havlicek, J., Roubalová, K. \& Flegr, J. (2005). Probable neuroimmunological link between Toxoplasma and cytomegalovirus infections and personality changes in the human host. BMC Infectious Diseases, 5(54):1-10.

Oskouei, M. M., Hamidi, F., Talebi, M., Farhoudi, M., Taheraghdam, A. A., Kazemi, T., . . Fallah, E. (2016a). The correlation between Toxoplasma gondii infection and Parkinson's disease: a casecontrol study. Journal of Parasitic Diseases, 40(3):872-876.

Oskouei, M. M., Hamidi, F., Talebi, M., Farhoudi, M., Taheraghdam, A. A., Kazemi, T., . . Fallah, E. (2016b). Toxoplasmosis and Alzheimer: can Toxoplasma gondii really be introduced as a risk factor in etiology of Alzheimer? Parasitology Research, 115(8):3169-3174.

Parlog, A., Schlüter, D. \& Dunay, I. R. (2015). Toxoplasma gondii-induced neuronal alterations. Parasite Immunology, 37(3):159-170.

Pedersen, M. G., Mortensen, P. B., Norgaard-Pedersen, B. \& Postolache, T. T. (2012). Toxoplasma gondii infection and self-directed violence in mothers. Archives of General Psychiatry, 69(11):11231130.

Pereira-Chioccola, V. L., Vidal, J. E. \& Su, C. (2009). Toxoplasma gondii infection and cerebral toxoplasmosis in HIV-infected patients. Future Microbiology, 4(10):1363-1379.

Prandovszky, E., Gaskell, E., Martin, H., Dubey, J. P., Webster, J. P. \& McConkey, G. A. (2011). The neurotropic parasite Toxoplasma gondii increases dopamine metabolism. PloS One, 6(9):e23866.

Roy, A., Karoum, F. \& Pollack, S. (1992). Marked reduction in indexes of dopamine metabolism among patients with depression who attempt suicide. Archives of General Psychiatry, 49(6):447-450.

Sales, O. P., Vianey, E. L., Bernardes, N., Silva, P. P., Oliveira, P. M. \& Avelino, S. C. (2010). Compulsão: como viver com essa rotina obsessiva. Journal of Health Sciencie Institute, 28(1):1316.

Sawa, A. \& Snyder, S. H. (2002). Schizophrenia: diverse approaches to a complex disease. Science, 296(5568):692-695.

Skallova, A., Kodym, P., Frynta, D. \& Flegr, J. (2006). The role of dopamine in Toxoplasma-induced behavioural alterations in mice: an ethological and ethopharmacological study. Parasitology, 133(5):525-535.

Sugden, K., Moffitt, T. E., Pinto, L., Poulton, R., Williams, B. S. \& Caspi, A. (2016). Is Toxoplasma gondii infection related to brain and behavior impairments in humans? Evidence from a populationrepresentative birth cohort. PLoS One, 11(2):e0148435.

Torrey, E. F., Bartko, J. J., Lun, Z.-R. \& Yolken, R. H. (2006). Antibodies to Toxoplasma gondii in patients with schizophrenia: a meta-analysis. Schizophrenia Bulletin, 33(3):729-736.

Torrey, E. F., Bartko, J. J. \& Yolken, R. H. (2012). Toxoplasma gondii and other risk factors for schizophrenia: an update. Schizophrenia Bulletin, 38(3):642-647.

Torrey, E. F., Rawlings, R. \& Yolken, R. H. (2000). The antecedents of psychoses: a case-control study of selected risk factors. Schizophrenia Research, 46(1):17-23.

Torrey, E. F. \& Yolken, R. H. (2003). Toxoplasma gondii and schizophrenia. Emerging Infectious Diseases, 9(11):1375-1380.

Webster, J. P., Lamberton, P. H. L., Donnelly, C. A. \& Torrey, E. F. (2006). Parasites as causative agents of human affective disorders? The impact of anti-psychotic, mood-stabilizer and anti-parasite medication on Toxoplasma gondii's ability to alter host behaviour. Proceedings of the Royal Society B: Biological Sciences, 273(1589):1023-1030.

Yan, C., Liang, L.-J., Zheng, K.-Y. \& Zhu, X.-Q. (2016). Impact of environmental factors on the emergence, transmission and distribution of Toxoplasma gondii. Parasites \& Vectors, 9(137):1-7.

Yereli, K., Balcioğlu, I. C. \& Özbilgin, A. (2006). Is Toxoplasma gondii a potential risk for traffic accidents in Turkey? Forensic Science International, 163(1-2):34-37. 
Yolken, R. H., Bachmann, S., Rouslanova, I., Lillehoj, E., Ford, G., Torrey, E. F. \& Schroeder, J. (2001). Antibodies to Toxoplasma gondii in individuals with first-episode schizophrenia. Clinical Infectious Diseases, 32(5):842-844.

Yolken, R. H. \& Torrey, E. F. (2008). Are some cases of psychosis caused by microbial agents? A review of the evidence. Molecular Psychiatry, 13(5):470-479.

Recebido: 14 de janeiro, 2019.

Aprovado: 6 de fevereiro, 2019.

Publicado: 1 de março, 2019

Licenciamento: Este artigo é publicado na modalidade Acesso Aberto sob a licença Creative Commons Atribuição 4.0 (CC-BY 4.0), a qual permite uso irrestrito, distribuição, reprodução em qualquer meio, desde que o autor e a fonte sejam devidamente creditados. 